University of Nebraska - Lincoln

DigitalCommons@University of Nebraska - Lincoln

Martin Centurion Publications

Research Papers in Physics and Astronomy

2015

\title{
Mega-electron-volt ultrafast electron diffraction at SLAC National Accelerator Laboratory
}

\author{
Stephen Weathersby \\ SLAC National Accelerator Laboratory, spw@slac.stanford.edu \\ G. Brown \\ SLAC National Accelerator Laboratory \\ Martin Centurion \\ University of Nebraska-Lincoln, martin.centurion@unl.edu \\ T. F. Chase \\ SLAC National Accelerator Laboratory \\ Ryan Coffee \\ SLAC National Accelerator Laboratory, coffee@slac.stanford.edu
}

See next page for additional authors

Follow this and additional works at: https://digitalcommons.unl.edu/physicscenturion

Part of the Atomic, Molecular and Optical Physics Commons

Weathersby, Stephen; Brown, G.; Centurion, Martin; Chase, T. F.; Coffee, Ryan; Corbett, Jeff; Eichner, J. P.; Frisch, J. C.; Fry, A. R.; Gühr, M.; Hartmann, Nick; Hast, Carsten; Hettel, R.; Jobe, R. K.; Jongewaard, E. N.; Lewandowski, J. R.; Li, R. K.; Lindenberg, A. M.; Makasyuk, Igor; May, J. E.; McCormick, D.; Nguyen, M. N.; Reid, Alexander; Shen, Xiaozhe; Sokolowski-Tinten, K.; Vecchione, Theodore; Vetter, Sharon; Wu, J.; Yang, Jie; Dürr, H. A.; and Wang, Xijie, "Mega-electron-volt ultrafast electron diffraction at SLAC National Accelerator Laboratory" (2015). Martin Centurion Publications. 23.

https://digitalcommons.unl.edu/physicscenturion/23

This Article is brought to you for free and open access by the Research Papers in Physics and Astronomy at DigitalCommons@University of Nebraska - Lincoln. It has been accepted for inclusion in Martin Centurion Publications by an authorized administrator of DigitalCommons@University of Nebraska - Lincoln. 


\section{Authors}

Stephen Weathersby, G. Brown, Martin Centurion, T. F. Chase, Ryan Coffee, Jeff Corbett, J. P. Eichner, J. C. Frisch, A. R. Fry, M. Gühr, Nick Hartmann, Carsten Hast, R. Hettel, R. K. Jobe, E. N. Jongewaard, J. R. Lewandowski, R. K. Li, A. M. Lindenberg, Igor Makasyuk, J. E. May, D. McCormick, M. N. Nguyen,

Alexander Reid, Xiaozhe Shen, K. Sokolowski-Tinten, Theodore Vecchione, Sharon Vetter, J. Wu, Jie Yang, H. A. Dürr, and Xijie Wang 


\title{
Mega-electron-volt ultrafast electron diffraction at SLAC National Accelerator Laboratory
}

\author{
S. P. Weathersby, ${ }^{1}$ G. Brown, ${ }^{1}$ M. Centurion, ${ }^{2}$ T. F. Chase, ${ }^{1}$ R. Coffee,${ }^{1}$ J. Corbett,${ }^{1}$ \\ J. P. Eichner, ${ }^{1}$ J. C. Frisch, ${ }^{1}$ A. R. Fry, ${ }^{1}$ M. Gühr, ${ }^{1}$ N. Hartmann,,${ }^{1}$ C. Hast, ${ }^{1}$ R. Hettel, ${ }^{1}$ \\ R. K. Jobe,${ }^{1}$ E. N. Jongewaard, ${ }^{1}$ J. R. Lewandowski, ${ }^{1}$ R. K. Li, ${ }^{1}$, a) A. M. Lindenberg, ${ }^{1}$ \\ I. Makasyuk,${ }^{1}$ J. E. May, ${ }^{1}$ D. McCormick, ${ }^{1}$ M. N. Nguyen, ${ }^{1}$ A. H. Reid, ${ }^{1}$ X. Shen, ${ }^{1}$ \\ K. Sokolowski-Tinten, ${ }^{3}$ T. Vecchione,${ }^{1}$ S. L. Vetter, ${ }^{1}$ J. Wu, ${ }^{1}$ J. Yang, ${ }^{2}$ H. A. Dürr, ${ }^{1}$ \\ and X. J. Wang ${ }^{1}$ \\ ${ }^{1}$ SLAC National Accelerator Laboratory, 2575 Sand Hill Road, Menlo Park, California 94025, USA \\ ${ }^{2}$ University of Nebraska-Lincoln, 855 N 16th Street, Lincoln, Nebraska 68588, USA \\ ${ }^{3}$ University of Duisburg-Essen, Lotharstrasse 1, 47048 Duisburg, Germany
}

(Received 9 May 2015; accepted 6 July 2015; published online 24 July 2015)

\begin{abstract}
Ultrafast electron probes are powerful tools, complementary to x-ray free-electron lasers, used to study structural dynamics in material, chemical, and biological sciences. High brightness, relativistic electron beams with femtosecond pulse duration can resolve details of the dynamic processes on atomic time and length scales. SLAC National Accelerator Laboratory recently launched the Ultrafast Electron Diffraction (UED) and microscopy Initiative aiming at developing the next generation ultrafast electron scattering instruments. As the first stage of the Initiative, a mega-electron-volt $(\mathrm{MeV}) \mathrm{UED}$ system has been constructed and commissioned to serve ultrafast science experiments and instrumentation development. The system operates at $120-\mathrm{Hz}$ repetition rate with outstanding performance. In this paper, we report on the SLAC MeV UED system and its performance, including the reciprocal space resolution, temporal resolution, and machine stability. (C) 2015 AIP Publishing LLC. [http://dx.doi.org/10.1063/1.4926994]
\end{abstract}

\section{INTRODUCTION}

Visualization of structural changes at atomic length and time scales establishes the connection between the structure, dynamics, and functionality, and paves the way for fully understanding, and ultimately, controlling energy and matter. ${ }^{1,2}$ To access the relevant length and time scales, probes with both short wavelengths ( $\lesssim 1 \AA$ ) and short pulse durations ( $\lesssim 100 \mathrm{fs})$ are required. Electrons and $\mathrm{x}$-rays are the most viable options for this challenging and highly rewarding task. ${ }^{3,4}$ The development of cutting-edge instruments, such as x-ray free-electron lasers (XFELs) ${ }^{5,6}$ ultrafast electron diffraction (UED) ${ }^{7-10}$ and microscopes ${ }^{11-15}$, has been vigorously pursued in the last few decades.

Electrons and $\mathrm{x}$-rays are complementary tools used to study material structures. ${ }^{3,4,16}$ While $\mathrm{x}$-rays primarily interact with electrons in matter, electrons are sensitive to both electrons and nuclei. Electrons have $10^{4}-10^{6}$ times larger scattering cross sections and therefore are the ideal choice to capture information from nanometer-scale or smaller samples. For a wide range of samples, the elastic mean-free-path of electrons matches well with the pumping depth by optical pulses, ${ }^{17}$ which further enhances the efficiency of electron probes in pump-probe experiments. Electrons also have $10^{3}$ times less radiation damage per elastic scattering event. ${ }^{16}$ Electrons are charged particles and can be easily manipulated by electromagnetic lenses and accelerating structures in a compact setup.

\footnotetext{
a) Author to whom correspondence should be addressed. Electronic mail: lrk@slac.stanford.edu
}

So far, most UED systems are based on kilo-electron-volt DC electron sources. These instruments have been improved over years and generated many remarkable science results.

The performance of UED can be tremendously boosted using mega-electron-volt (MeV) electron sources, i.e., radiofrequency (rf) photoinjectors. ${ }^{18,19}$ The accelerating field in an rf photoinjector normally exceeds $100 \mathrm{MV} / \mathrm{m}$, which is much higher than the 10-20 MV/m level in DC photoinjectors, and allows the extraction of much brighter electron beams from the cathode, minimizing emittance and bunch length growth due to the space charge effects. The MeV final energy very effectively suppresses space charge effects, which scale as $\gamma^{3}$, where $\gamma$ is the Lorentz factor; thus, the beam brightness can be essentially preserved from the electron source to the detector. Using $\mathrm{MeV}$ electrons as the probe also naturally solves the velocity mismatch issue where sub-relativistic $\mathrm{keV}$ electrons lag behind the optical pump pulses, ${ }^{20,21}$ which is critical to reach sub-100 fs temporal resolution for gas phase samples. An rf bunching cavity can be used in both $\mathrm{keV}^{22-24}$ and $\mathrm{MeV}$ $\mathrm{UEDs}^{25,26}$ to compress the electron beam and achieve higher temporal resolution. With this scheme, the advantage of $\mathrm{MeV}$ UED is even more significant. The relative energy spread of electron beams translates to relative broadening of diffraction spots or rings. To maintain the relative energy spread at the tolerable level, a much larger absolute energy spread is allowed in MeV UED. For a given normalized longitudinal emittance, in principle much shorter pulse durations can be generated in MeV UED.

Since the first experimental demonstration of electron diffraction using sub-picosecond, $\mathrm{MeV}$ beams from an rf 
photoinjector at SLAC in 2006, ${ }^{27}$ intensive R\&D efforts have been devoted to improve the performance of the MeV UED system, including its reciprocal space resolution, temporal resolution, and machine stability. ${ }^{10,26,28-37}$ Photoinjectors were originally optimized for operating at $100 \mathrm{~s}$ of $\mathrm{pC}$ or higher bunch charge, while for MeV UED purposes, a few $\mathrm{pC}$ or lower per pulse is preferred. A series of new techniques for the generation, control, and characterization of low charge high brightness electron beams has been developed. Based on these achievements, it is become feasible to construct an MeV UED system capable of productively generating high quality data for ultrafast sciences.

SLAC recently launched the UED/ultrafast electron microscopy (UEM) Initiative ${ }^{38}$ aimed at developing the world's leading ultrafast electron scattering instruments. These instruments are complementary to the Linac Coherent Light Source (LCLS) XFEL, providing a more complete picture of ultrafast processes in chemical, biological, and other complex systems. The first stage of the Initiative was to construct an $\mathrm{MeV}$ UED system. MeV UED and LCLS share many common key technologies since the electron source of LCLS is also an rf photoinjector. The high stability rf power source and the high precision laser-rf timing system developed for the LCLS are of critical importance to reduce the time-of-arrival jitter between the pump laser and electron probe pulses in order to eventually break the 100-fs temporal resolution barrier. The $120-\mathrm{Hz}$ repetition rate of the LCLS-type photoinjector enables efficient acquisition of data having high signal-to-noise ratio data within a reasonable exposure time. The SLAC MeV UED system can handle both solid state and gas phase samples using two dedicated sample chambers. These unique features allow the SLAC MeV UED system to directly serve ultrafast science experiments with outstanding performance. In this paper, we will report on the newly commissioned SLAC MeV UED system and the characterization of the system performance, including the reciprocal space resolution, temporal resolution, and stability of key machine parameters.

\section{MeV UED SYSTEM AT SLAC}

The MeV UED system is housed in the Accelerator Structure Test Area (ASTA) at SLAC. A schematic of the UED beam line is shown in Fig. 1. Key components of the system include a LCLS-type photocathode rf gun, a sample chamber, a high efficiency electron detector, an ultra-stable rf power source, a Ti:Sapphire laser, and a laser-rf timing system.
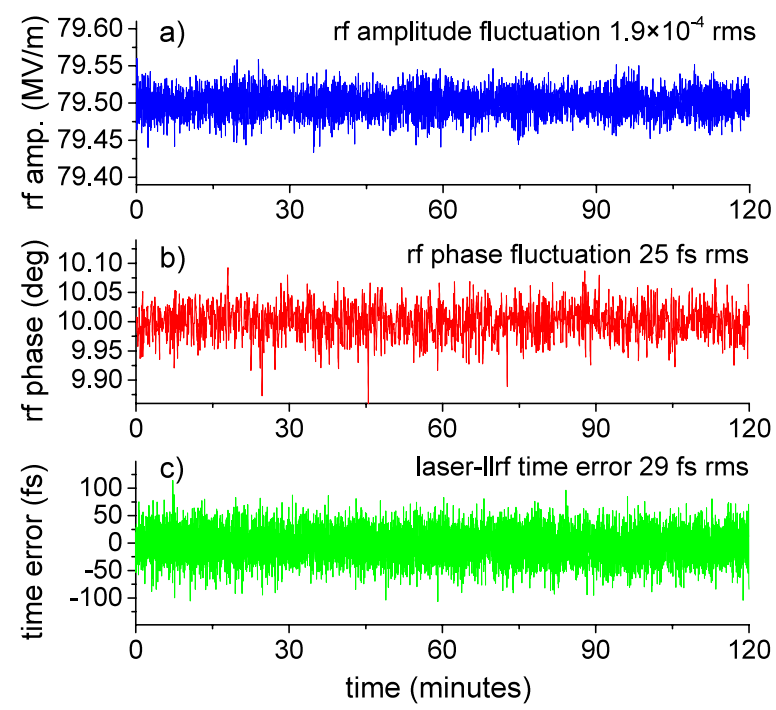

FIG. 2. The (a) amplitude and (b) phase fluctuations of the rf field in the gun, and (c) the time error between the laser and the low-level rf system.

The LCLS-type photocathode rf gun is identical to the one used for the LCLS injector. ${ }^{39,40}$ The rf gun is powered by a pulse-forming-network- (PFN-) based modulator and a 50MW S-band klystron. The modulator was upgraded by adding a top-off charging power supply to the PFN that stabilizes the output voltage to be better than $50 \mathrm{ppm} .{ }^{41}$ A Phase and Amplitude Detector (PAD) unit ${ }^{42,43}$ monitors the rf signal in the gun. A feedback loop stabilizes the rf phase and amplitude in the gun relative to a low-level reference rf signal by adjusting the input to the klystron using a Phase and Amplitude Controller (PAC) unit. ${ }^{44}$ The typical values of the rf amplitude and phase stability of the gun field are $2 \times 10^{-4}$ rms and 25 fs rms over hours, measured by rf antennas on the gun body, as shown in Figs 2(a) and 2(b). To achieve low time-of-arrival jitter between the pump laser and probe electron pulses, the laser system also needs to be precisely synchronized with the rf signal. A low-cost, high-reliability femtosecond timing system developed for LCLS ${ }^{45}$ was implemented for the MeV UED system. The measured in-loop timing error between the laser and the low-level rf signal is shown in Fig. 2(c). This in-loop value is in good agreement with that measured by a phase noise analyzer using an independent detector and electronics.

A kilohertz, 5-mJ Ti:Sapphire laser system is used for both generating electron beams from the photocathode and for pumping the samples. A small fraction (10\%) of the IR output is used for UV generation. The UV pulse is imaged

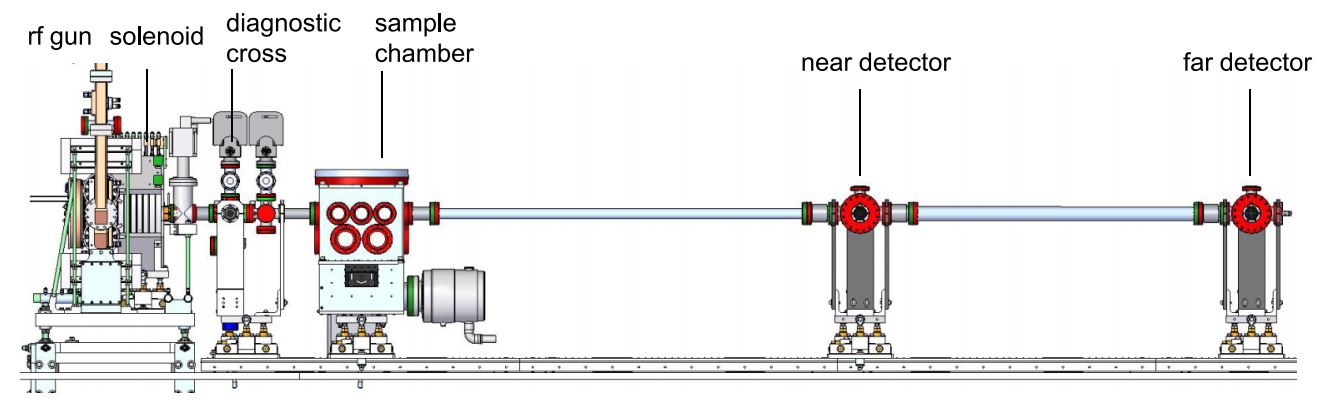

FIG. 1. Schematic of the MeV UED beam line at SLAC's ASTA facility. 

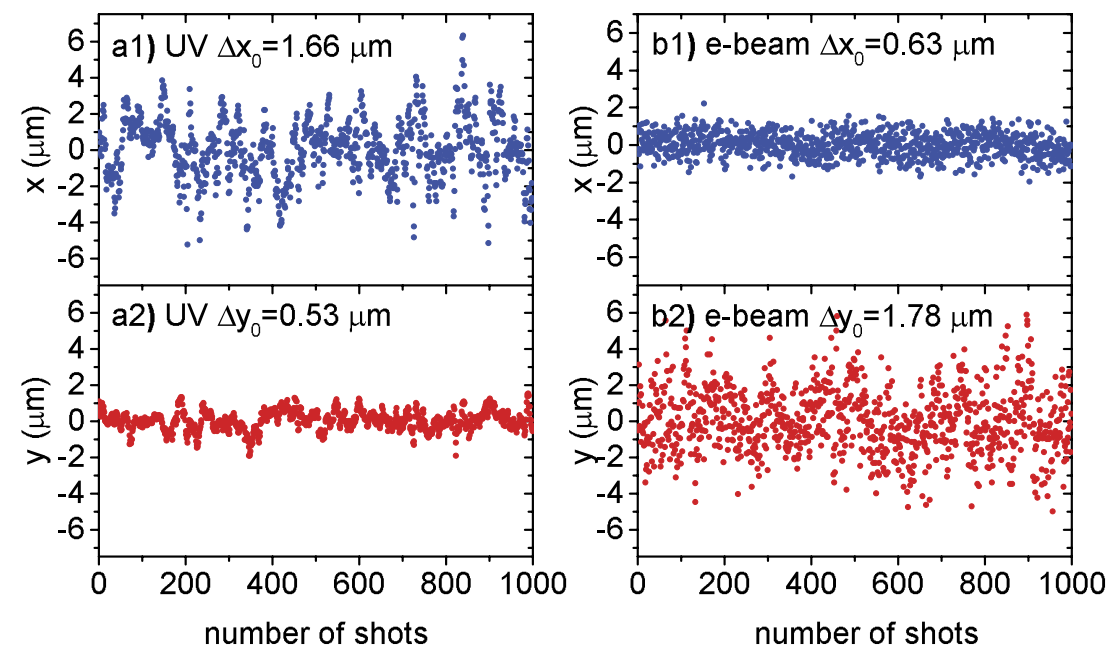

FIG. 3. ((a1) and (a2)) Centroid stabilities of the UV laser on the virtual cathode screen are $1.66 \mu \mathrm{m}$ rms and $0.53 \mu \mathrm{m}$ rms, corresponding to $4.2 \%$ and $1.4 \%$ of the rms spot size in $x$ and $y$ directions, respectively. ((b1) and (b2)) Centroid stabilities of the electron beam on the diagnostic screen $(z=55.8 \mathrm{~cm})$ are $0.63 \mu \mathrm{m}$ rms and $1.78 \mu \mathrm{m} \mathrm{rms}$, corresponding to $1.1 \%$ and $3.0 \%$ of the rms spot size in $x$ and $y$ directions, respectively.

from a grating surface onto the photocathode with an $8: 1$ demagnification and illuminates the cathode at a $70^{\circ}$ angle of incidence. The grating surface is parallel to the photocathode, and the UV pulse-front is also parallel to the cathode surface to minimize the electron bunch length. The UV beam profile and position on the cathode are monitored by a virtual cathode camera. The pump path includes a $30-\mathrm{cm}$ translation stage for adjusting the delay between pump and probe pulses. Since adequate pump laser energy is available, the pump spot size is usually 3-4 times larger than the probe size to ensure uniform pumping of the sample. Relay imaging was set up from the laser amplifier exit to the photocathode and to the sample location, respectively, for optimal laser pointing stability. In Fig. 3, we show that the stability of the UV centroid on the virtual cathode camera is at the $1-\mu \mathrm{m} \mathrm{rms} \mathrm{level.} \mathrm{The} \mathrm{centroid} \mathrm{of}$ electron beams measured at the diagnostic screen $55.8 \mathrm{~cm}$ from the photocathode is clearly dominated by the UV pointing jitter. Note in Fig. 3 the focusing solenoid rotates the electron beam by $86^{\circ}$ along the beam axis.

The solid state sample chamber hosts a sample stage with 4-axis motion control, i.e., 1-in. translation in $x$ and $y$ directions and $\pm 90^{\circ}$ rotation along $x$ and $y$ axes. An in-vacuum mirror is installed in the sample chamber to enable laserpumping the sample from the front surface with a 50-mrad angle of incidence. Without pulse-front-tilt correction, the small incident angle introduces $<20$-fs rms broadening of the temporal resolution for a $400-\mu \mathrm{m}$ diameter probe size. The sample holder is $1 \times 1 \mathrm{in}$. in dimension and can accommodate 10-20 samples of various sizes (standard TEM grids or square $\mathrm{SiN}$ windows), as well as a scintillator screen for identifying the transverse overlap of the probe electron beam and the pump laser beams, and a fast photodiode to measure the coarse timing between the pump and probe pulses with $\sim 100$-ps precision. A shadowgraph of laser-generated ultrafast plasma from TEM grids is used to determine the time-zero with $\sim 100$-fs accuracy. ${ }^{46-50}$

The electron detector consists of a $\mathrm{P} 43$ phosphor screen perpendicular to the beam path, $\mathrm{a} 45^{\circ}$ in-vacuum mirror, a large aperture coupling lens, and an Andor iXon Ultra 888 electron multiplying charge-coupled device camera (EMCCD). ${ }^{51}$ The phosphor screen is 50-100 $\mu \mathrm{m}$ in thickness with an optimized grain size for maximum efficiency. A $40-\mathrm{mm} \mathrm{f} / 0.85$ lens is used to image the phosphor screen. The point-spread-function of the detector is $85 \mu \mathrm{m} \mathrm{rms}$.

The typical machine and beam parameters are summarized in Table I. The system can operate stably over an extended period of time ( $>24 \mathrm{~h}$ ) allowing systematic measurement of the dynamic processes.

\section{RECIPROCAL SPACE RESOLUTION}

High reciprocal space resolution, or $s$-resolution, allows unambiguous identification of the ring or spot features in diffraction patterns, and tracking changes of their intensity,

TABLE I. Typical machine and beam parameters of the MeV UED system.

\begin{tabular}{lc}
\hline \hline Parameters & Values \\
\hline Repetition rate & $120 \mathrm{~Hz}$ \\
Gun gradient & $79.5 \mathrm{MV} / \mathrm{m}$ \\
Launching phase & $10^{\circ}$ \\
Solenoid strength & $0.314 \mathrm{kG}-\mathrm{m}$ \\
UV spot size, rms & $40 \mu \mathrm{m}$ \\
UV pulse duration, FWHM & $60 \mathrm{fs}$ \\
UV energy stability, rms & $2.5 \%$ \\
Initial beam charge & $75 \mathrm{fC}$ \\
Intrinsic emittance & $0.5 \mathrm{mrad}$ \\
Collimator diameter & $500 \mu \mathrm{m}$ \\
(z=55.8 cm) & \\
At the sample (z=1.16 m) & \\
Beam charge & $60 \mathrm{fC}$ \\
Beam size (diameter) & $400 \mu \mathrm{m}$ \\
Normalized emittance & $18 \mathrm{~nm}-\mathrm{rad}$ \\
Bunch length, rms & $102 \mathrm{fs}$ \\
Kinetic beam energy & $3.68 \mathrm{MeV}$ \\
Relative energy spread, rms & $6.6 \times 10^{-4}$ \\
IR pump spot size (diameter) & $1.5 \mathrm{~mm}$ \\
IR pump pulse duration, FWHM & $60 \mathrm{fs}$ \\
\hline \hline
\end{tabular}


position, and width with high precision. The momentum transfer $s$ is defined as $s=2 \pi \frac{\theta}{\lambda}$, where $\theta$ is the scattering angle at the sample, $\lambda=\frac{h}{\gamma \beta m_{0} c_{0}}$ is the de Broglie wavelength of the probe electrons, $\gamma$ is the Lorentz factor, $\beta$ is the normalized speed, $m_{0}$ is the electron rest mass, and $c_{0}$ is the speed of light. The $s$-resolution can be written as $\Delta s=2 \pi \frac{\Delta r / L}{\lambda}$, where $\Delta r$ is the rms width of the diffraction spot or ring and $L$ is the distance between the sample and the detector.

The $s$-resolution can also be written as $\Delta s=2 \pi \frac{\sigma_{x^{\prime}}}{\lambda}$, assuming the width of the diffraction spots or rings is dominated by the intrinsic beam divergence $\sigma_{x^{\prime}}$ at the sample. The assumption is valid when the solenoid is tuned to focus the electron beam onto the detector, space charge effects induced growth of spot or ring width from the sample to the detector is negligible, and the point-spread-function of the detector is much smaller than the electron beam size. The intrinsic beam divergence is defined as the rms width of the $x^{\prime}$ distribution in the $x-x^{\prime}$ phase space after the linear correlation is removed; hence the normalized beam emittance is $\epsilon_{n}=\gamma \beta \sigma_{x} \sigma_{x^{\prime}}$, where $\sigma_{x}$ is the rms spot size at the sample. Then the $s$-resolution can be expressed as

$$
\Delta s=\frac{2 \pi}{\lambda_{e}} \frac{\epsilon_{n}}{\sigma_{x}},
$$

where $\lambda_{e}$ is the Compton wavelength of electrons. For a given probe size, the $s$-resolution is defined by the beam emittance. Above discussion has ignored the contribution to the $s$-resolution from sample conditions. The grain size, defects, impurities, etc., of real samples will result in larger $\Delta r$ and hence degrades the $s$-resolution.

In the current MeV UED setup, the solenoid is the only focusing element and is tuned to deliver the sharpest diffraction features to the detector. The probe size is controlled by a collimator located $55.8 \mathrm{~cm}$ from the cathode. Different collimator sizes can be used to match the available sample area. In Fig. 4, we show the measured and simulated beam spot size with $500 \mu \mathrm{m}, 200 \mu \mathrm{m}$, and $100 \mu \mathrm{m}$ collimator diameters. The bunch charge, probe size, beam emittance, and $s$-resolution for each case is summarized in Table II. The bunch charge and probe size were measurement results. The emittance values were extracted from simulations, and the $s$-resolutions were calculated using Eq. (1).

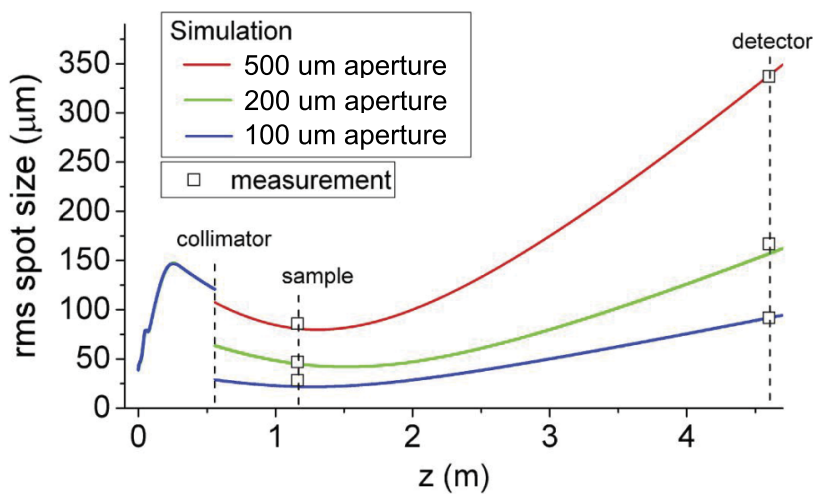

FIG. 4. (solid lines) Simulated electron beam spot size along the beam line for $500 \mu \mathrm{m}$ (red), $200 \mu \mathrm{m}$ (green), and $100 \mu \mathrm{m}$ (blue) collimator diameters. The squares represent measurement results.
TABLE II. The bunch charge $Q$, probe size $\sigma_{x}$, normalized beam emittance $\epsilon_{n}$, and $s$-resolution $\Delta s$ with different collimator sizes.

\begin{tabular}{lcccc}
\hline \hline $\begin{array}{l}\text { Collimator } \\
\text { diameter }(\mu \mathrm{m})\end{array}$ & $Q(\mathrm{fC})$ & $\sigma_{x}(\mu \mathrm{m})$ & $\epsilon_{n}(\mathrm{~nm}-\mathrm{rad})$ & $\Delta s\left(\AA^{-1}\right)$ \\
\hline 500 & 61 & 86 & 18 & 0.054 \\
200 & 11 & 47 & 7.1 & 0.039 \\
100 & 1.4 & 29 & 2.1 & 0.019 \\
\hline \hline
\end{tabular}

\section{TEMPORAL RESOLUTION}

The temporal resolution $\tau$ of the MeV UED system is

$$
\tau=\sqrt{\tau_{e}^{2}+\tau_{p h}^{2}+\tau_{\mathrm{TOA}}^{2}+\tau_{\mathrm{VM}}^{2}},
$$

where $\tau_{e}$ and $\tau_{p h}$ are the pulse durations of the probe and pump pulses, respectively, $\tau_{\mathrm{TOA}}$ is the time-of-arrival jitter between the pump and probe pulses, and $\tau_{\mathrm{VM}}$ is the velocity mismatch..$^{20,21}$

In an rf gun-based MeV UED system, $\tau_{\mathrm{TOA}}$ is due to the rf amplitude fluctuation and the phase timing error relative to the laser system..$^{52}$ In the SLAC MeV UED system, the timing error between the laser and the low-level rf system is $<30 \mathrm{fs}$ $\mathrm{rms}$, and the timing error between the same low-level rf signal and the high power rf field is $<30 \mathrm{fs}$ rms, as shown in Fig. 2. Thus, the timing jitter between the laser and the gun rf field is $45 \mathrm{fs}$ rms. 45 -fs rms jitter of the launching phase translates to 30 -fs rms error in the time-of-flight from the cathode to the sample position at $80-\mathrm{MV} / \mathrm{m}$ gun gradient and $10^{\circ}$ launching phase. Under the same gun operating condition, $2 \times 10^{-4} \mathrm{rms}$ rf amplitude jitter will contribute 15 -fs rms time-of-flight jitter. Neglecting the correlation between these terms, the upper limit of $\tau_{\mathrm{TOA}}$ is estimated to be $<50 \mathrm{fs} \mathrm{rms}$.

The pulse duration of the electron probe beam is dominated by the space charge-induced energy chirp. The electron beam is generated with a pancake shape and high charge density at the cathode. The longitudinal space charge forces pushes the head of the beam to a higher energy, travelling faster than the beam tail. The space charge induced bunch lengthening effect depends on the initial charge density and the extraction field $E_{\text {acc }} \sin \phi$, where $E_{\text {acc }}$ is the gun gradient and $\phi$ is the launching phase. We chose $10^{\circ}$ launching phase to provide a balance between the space charge lengthening and the rf compression effect in the gun ${ }^{53}$ and that generates the shortest bunch length at the sample. In Fig. 5(a), we show the simulated bunch length at the sample position as a function of the initial beam charge.

In experiment, we measured the ultrafast laser induced structural changes of the a 25 -nm-thick $\mathrm{Bi}(111)$ thin film using 20 -fC and $80-\mathrm{fC}$ bunch charges. Other beam parameters were kept identical as shown in Table I. In Fig. 5(b), we show the temporal evolution of the normalized intensity of the (410) ring. A simple erfc function fit of the 20-fC data yields a time constant of $217 \mathrm{fs}$ rms. We can also model the traces using a convolution between an exponential decay and a Gaussian distribution, which are assumed to be the intrinsic response of the sample and the temporal resolution of the instrument, respectively. Assuming the intrinsic response 

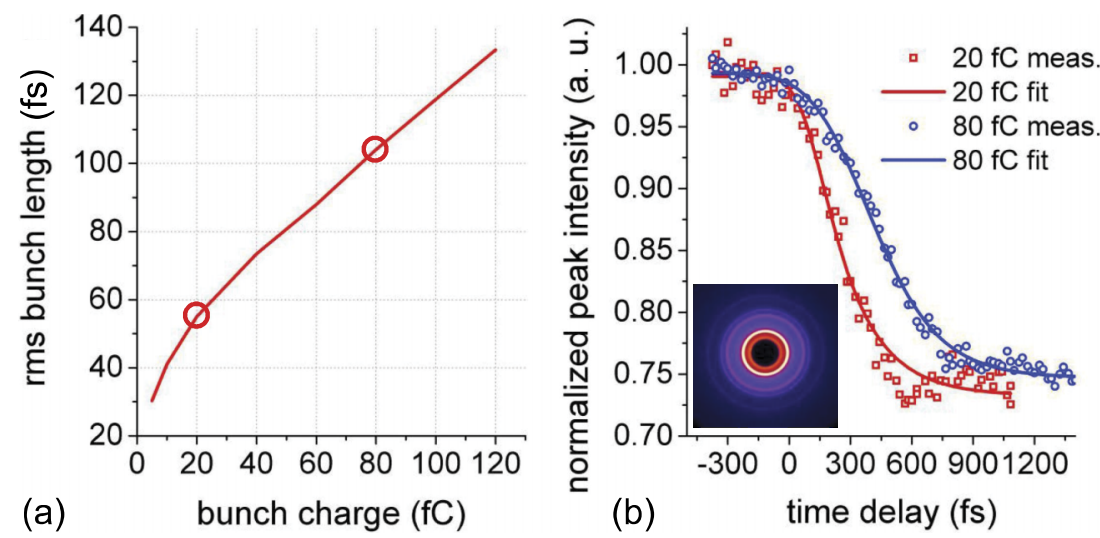

FIG. 5. (a) Simulated electron beam bunch length at the sample position as a function of initial beam charge. (b) Time-dependent intensity evolution of the (410) ring of 25 -nm-thick Bi(111) sample with 20 -fC and 80 -fC beam charges.

of the sample 54,55 is $150 \pm 50 \mathrm{fs} \mathrm{rms}$, and then the temporal resolution of the instrument is $105 \pm 27 \mathrm{fs}$ rms.

A deflecting cavity will be installed to directly measure the electron beam bunch length. Time-stamping techniques similar to those developed for ultrashort $\mathrm{X}$-ray pulses ${ }^{56-60}$ will be explored to characterize $\tau_{\mathrm{TOA}}$ of the MeV UED system.

\section{GAS PHASE MeV UED}

Electrons are the ideal choice to probe gas phase samples due to their many orders of magnitude larger scattering cross section compared to X-rays. Also, electrons, especially $\mathrm{MeV}$ electrons, have sub-picometer de Broglie wavelength and thus provide a much larger $s$-range in the diffraction pattern. With $\mathrm{MeV}$ electrons, the velocity mismatch issue is essentially eliminated compared to the low velocity keV UED case. Thus, there is promise that the 100-fs time resolution barrier can be broken using MeV UED, enabling making movies of ultrafast chemical reactions.

One of the major technical challenges for the gas phase $\mathrm{MeV}$ UED is to manage the extremely different vacuum levels at different parts of the beam line-the gas phase sample chamber will operate at the $5 \times 10^{-5}$ Torr level while the photocathode rf gun has to be maintained at $5 \times 10^{-10}$ Torr or better. The solution in our case was to employ a 50-nm-thin SiN membrane to separate the two sections while preserving the probe beam quality at a reasonable level.

According to the kinetic scattering theory, the number of scattering events $n$ will follow the Poisson distribution $P(n)$ $=(t / \tau)^{n} e^{-t / \tau} / n !$, where $t$ is the thickness of the scattering membrane and $\tau$ is the scattering mean free path. $P(0)$ is the fraction of electrons that will go through the SiN membrane without scattering. These electrons, called direct beam, retain the low divergence and will contribute to the diffraction pattern. We measured the transmission of the $\mathrm{MeV}$ electron beams through the $\mathrm{SiN}$ membranes of different thicknesses, as shown in Fig. 6. The distance between the $\mathrm{SiN}$ windows and the detector is $3.44 \mathrm{~m}$. The measurement results are in good agreement with the Poisson distribution. The measured beam size confirmed that the direct beam has the same divergence hence emittance as the incident beam and is adequate for diffraction. The direct beam size was decreasing with the increase of the SiN membrane thickness because of the loss of the transmitted charge and hence the reduction in space
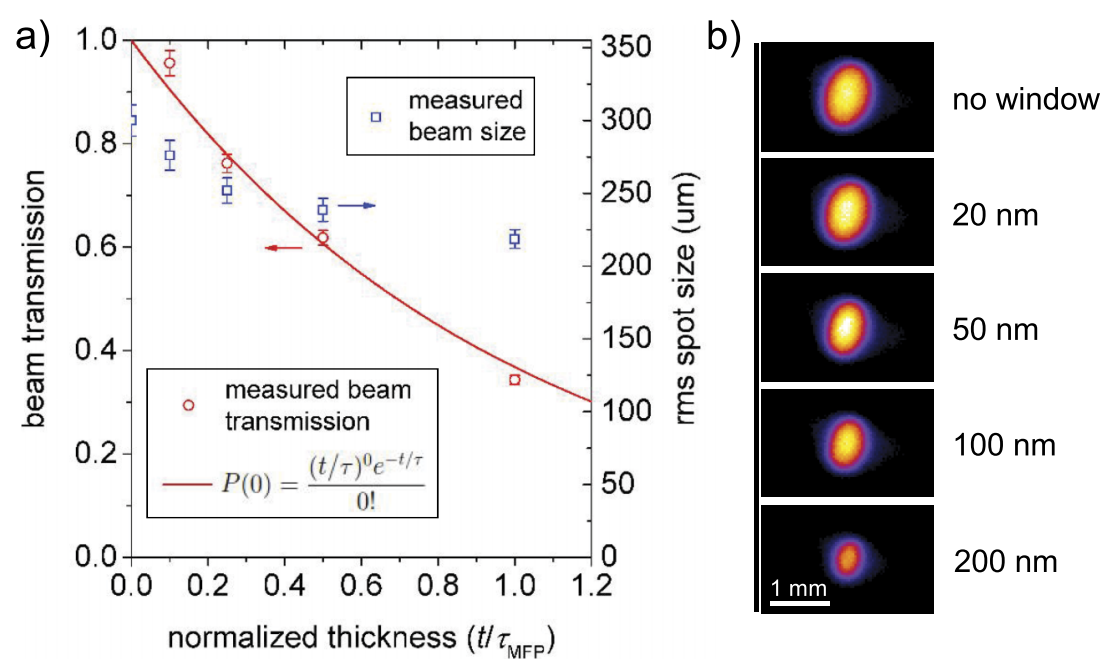

FIG. 6. (a) Transmission and spot size of the direct beams through SiN membranes of different thicknesses. The membrane thickness is normalized by the scattering mean-free-path (MFP). (b) The electron beam profiles measured at $3.44 \mathrm{~m}$ downstream of the SiN membranes. 

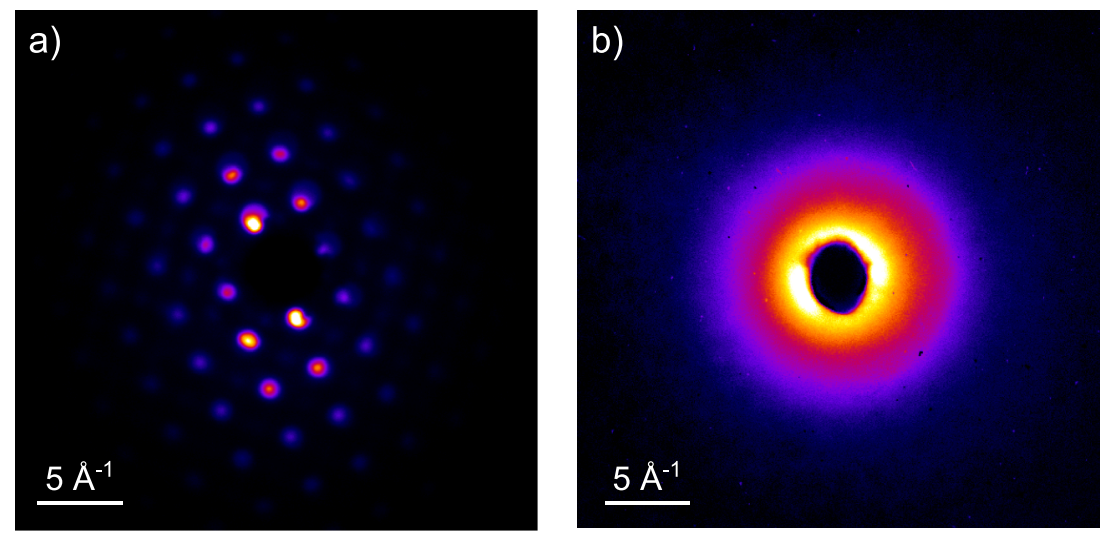

FIG. 7. Diffraction patterns of (a) single crystal gold and (b) $N_{2}$ samples recorded in the gas phase MeV UED configuration.

charge effects. 50-nm thickness was chosen as a good balance between the transmission and the mechanical robustness of the membrane.

Nevertheless, the scattering from the SiN membrane, being background noise, could still be much stronger than the signal from gas phase samples which are typically of very low density. A second collimator of $200-\mu \mathrm{m}$ diameter was installed at $z=1.44 \mathrm{~m}$, in addition to the first collimator at $z=55.8 \mathrm{~cm}$. The SiN membrane and the gas nozzle are located at $z=74 \mathrm{~cm}$ and $1.56 \mathrm{~m}$, respectively. The second collimator was used to limit the angular range of the SiN scattering. On the diffraction detector located $2.1 \mathrm{~m}$ after the second collimator, the $\mathrm{SiN}$ scattering can only illuminate an area of $<1 \mathrm{~mm}$ from the beam axis, assuming a $400-\mu \mathrm{m}$ diameter spot size at the $\mathrm{SiN}$ membrane. We confirmed in experiment that the above beam line configuration provides a low noise range between 1 and $15 \AA^{-1}$ on the detector.

In Figs. 7(a) and 7(b), we show the diffraction patterns of single crystal gold and nitrogen $\left(N_{2}\right)$ samples, respectively. The $N_{2}$ sample density is $2 \times 10^{17} \mathrm{~cm}^{-3}$ with an effective thickness of $300 \mu \mathrm{m}$. The total number of incident electrons is $2 \times 10^{9} e^{-}$. With $10-\mathrm{fC}$ bunch charge and $120 \mathrm{~Hz}$ repetition rate, a total exposure time of $300 \mathrm{~s}$ is required. A planned upgrade of the system is to install an rf bunching cavity, which can longitudinally compress the electron beams by velocity bunching while preserving the transverse emittance. . $2,23,26,61-63$ This upgrade will allow much higher bunch charge and reduce the exposure time by more than one order of magnitude.

\section{SUMMARY AND OUTLOOK}

In summary, an MeV UED system was constructed and commissioned at SLAC. The system operates at $120-\mathrm{Hz}$ repetition rate with outstanding temporal resolution, reciprocal space resolution, and machine stability. The success of the system benefited tremendously from the worldwide R\&D effort on MeV UED that has taken place in the last decade. SLAC's technical strength in providing a complete set of hardware and technologies developed for the photoinjector system was critical for achieving the machine performance.

The SLAC MeV UED system has been used to study several ultrafast dynamic processes. These include a detailed study of electron-phonon coupling in Bismuth films as a function of film thickness and laser fluence; a measurement of the lattice expansion of free-standing FePt nanoparticles-a material used in next generation magnetic storage; structural response and deformations of single-layer transition metal dichalcogenide $\mathrm{MoS}_{2}$ following optical excitation; and thermal diffuse scattering and phonon equilibration in single crystal gold, etc. These results demonstrate that MeV UED is capable of serving ultrafast science experiments with high data quality and high productivity.

Future upgrades of the SLAC MeV UED system will further improve the probe electron beam, as well as enhance the sample and pump capabilities. A strong lens will be installed to reduce the probe size from $100 \mu \mathrm{m}$ to a few $\mu \mathrm{m}$ and eventually to sub- $\mu \mathrm{m}$ for nano-UED, ${ }^{64}$ which will greatly ease the technical challenges in sample preparation, and moreover, allow study of single crystalline domains. An rf bunching cavity will be used to compress the pulse duration to the 10-fs level. Combined with new time-of-arrival control and time-stamping techniques, it is possible to reach 20 -fs temporal resolution. Cooling and heating capabilities will be integrated for solid state samples to study, for example, strongly correlated systems and martensitic phase transitions. The SiN membrane based vacuum separation technique will also allow liquid jet samples to be studied. A few $\mu$ m probe size will be well suited to look into the dynamics happening in liquid cells ${ }^{65} \mathrm{THz}$ and $\mathrm{x}$-ray pumps, particularly the extremely high intensity THz source at SLAC's FACET facility ${ }^{66}$ and $\mathrm{x}$ ray pulses at the LCLS, will excite materials into exotic new states that, combined with MeV UED probes, will provide opportunities for ground breaking science.

\section{ACKNOWLEDGMENTS}

The authors are grateful to their SLAC colleagues for the strong management and technical support during the planning, design, installation, and commissioning stages of the $\mathrm{MeV}$ UED system. This work was supported in part by the U.S. Department of Energy Contract No. DE-AC02-76SF00515 and the SLAC UED/UEM Initiative Program Development Fund. J. Yang and M. Centurion were partially supported by the U.S. Department of Energy Office of Science, Office of Basic Energy Sciences under Award No. DE-SC0003931. 
1"Directing matter and energy: Five challenges for science and the imagination," A Report from the Basic Energy Sciences Advisory Committee, 2007, (http://science.energy.gov/ /media/bes/pdf/reports/files/gc_rpt.pdf.

2"Future of electron scattering and diffraction," Report of the Basic Energy Sciences Workshop on the Future of Electron Scattering and Diffraction, 2014, (http://science.energy.gov/ /media/bes/pdf/reports/files/ Future_of_Electron_Scattering.pdf.

${ }^{3}$ M. Chergui and A. H. Zewail, ChemPhysChem 10, 28 (2009).

${ }^{4}$ R. J. D. Miller, Science 343, 1108 (2014).

${ }^{5}$ P. Emma et al., Nat. Photonics 4, 641 (2010).

${ }^{6}$ T. Ishikawa et al., Nat. Photonics 6, 540 (2010).

${ }^{7}$ W. E. King, G. H. Campbell, A. Frank, B. Reed, J. F. Schmerge, B. J. Siwick, B. C. Stuart, and P. M. Weber, J. Appl. Phys. 97, 111101 (2005).

${ }^{8}$ A. H. Zewail, Annu. Rev. Phys. Chem. 57, 65 (2006).

${ }^{9}$ G. Sciaini and R. J. D. Miller, Rep. Prog. Phys. 74, 096101 (2011).

${ }^{10} \mathrm{P}$. Musumeci and R. K. Li, in ICFA Beam Dynamics Newsletter No. 59, edited by J. M. Byrd and W. Chou (International Committee for Future Accelerators, 2012), pp. 13-33.

${ }^{11}$ B. W. Reed, M. R. Armstrong, N. D. Browning, G. H. Campbell, J. E. Evans, T. LaGrange, and D. J. Masiel, Microsc. Microanal. 15, 272 (2009).

${ }^{12}$ A. H. Zewail, Science 328, 187 (2010).

${ }^{13}$ A. H. Zewail and J. M. Thomas, 4D Electron Microscopy: Imaging in Space and Time (Imperial College Press, London, 2010).

${ }^{14}$ N. D. Browning, G. H. Campbell, J. E. Evans, T. B. LaGrange, K. L. Jungjohann, J. S. Kim, D. J. Masiel, and B. W. Reed, Handbook of Nanoscopy (Wiley-VCH Verlag GmbH \& Co. KGaA, 2012), Chap. 9.

${ }^{15}$ D. J. Flannigan and A. H. Zewail, Acc. Chem. Res. 45, 1828 (2012).

${ }^{16}$ R. Henderson, Q. Rev. Biophys. 28, 171 (1995).

${ }^{17}$ S. D. Brorson, J. G. Fujimoto, and E. P. Ippen, Phys. Rev. Lett. 59, 1962 (1987).

${ }^{18}$ X. J. Wang, Z. Wu, and H. Ihee, in Proceedings of 2003 Particle Accelerator Conference (IEEE, Portland, OR, USA, 2003), p. 420.

${ }^{19}$ X. J. Wang, D. Xiang, T. K. Kim, and H. Ihee, J. Korean Phys. Soc. 48, 390 (2006).

${ }^{20}$ J. C. Williamson and A. H. Zewail, Chem. Phys. Lett. 209, 10 (1993).

${ }^{21}$ P. Zhang, J. Yang, and M. Centurion, New J. Phys. 16, 083008 (2014).

${ }^{22}$ T. van Oudheusden, E. F. de Jong, S. B. van der Geer, W. P. E. M. O. 't Root, O. J. Luiten, and B. J. Siwick, J. Appl. Phys. 102, 093501 (2007).

${ }^{23}$ T. van Oudheusden, P. L. E. M. Pasmans, S. B. van der Geer, M. J. de Loos, M. J. van der Wiel, and O. J. Luiten, Phys. Rev. Lett. 105, 264801 (2010).

${ }^{24}$ R. P. Chatelain, V. R. Morrison, C. Godbout, and B. J. Siwick, Appl. Phys. Lett. 101, 081901 (2012).

${ }^{25}$ J. H. Han, Phys. Rev. Spec. Top.-Accel. Beams 14, 050101 (2011).

${ }^{26}$ R. K. Li, P. Musumeci, H. A. Bender, N. S. Wilcox, and M. Wu, J. Appl. Phys. 110, 074512 (2011).

${ }^{27}$ J. B. Hastings, F. M. Rudakov, D. H. Dowell, J. F. Schmerge, J. D. Cardoza, J. M. Castro, S. M. Gierman, H. Loos, and P. M. Weber, Appl. Phys. Lett. 89, 184109 (2006).

${ }^{28}$ P. Musumeci, J. T. Moody, and C. M. Scoby, Ultramicroscopy 108, 1450 (2008).

${ }^{29}$ R. K. Li, C. X. Tang, Y. C. Du, W. H. Huang, Q. Du, J. R. Shi, L. X. Yan, and X. J. Wang, Rev. Sci. Instrum. 80, 083303 (2009).

${ }^{30}$ R. K. Li, W. H. Huang, Y. C. Du, L. X. Yan, Q. Du, J. R. Shi, J. F. Hua, H. B. Chen, T. B. Du, H. S. Xu, and C. X. Tang, Rev. Sci. Instrum. 81, 036110 (2010).

${ }^{31}$ P. Musumeci, J. T. Moody, C. M. Scoby, M. S. Gutierrez, H. A. Bender, and N. S. Wilcox, Rev. Sci. Instrum. 81, 013306 (2010).

${ }^{32}$ P. Musumeci, J. T. Moody, C. M. Scoby, M. S. Gutierrez, and M. Westfall, Appl. Phys. Lett. 97, 063502 (2010).

${ }^{33}$ P. Musumeci, J. T. Moody, C. M. Scoby, M. S. Gutierrez, M. Westfall, and R. K. Li, J. Appl. Phys. 108, 114513 (2011).
${ }^{34}$ Y. Murooka, N. Naruse, S. Sakakihara, M. Ishimaru, J. Yang, and K. Tanimura, Appl. Phys. Lett. 98, 251903 (2011).

${ }^{35}$ P. Zhu, Y. Zhu, Y. Hidaka, L. Wu, J. Cao, H. Berger, J. Geck, R. Kraus, S. Pjerov, Y. Shen, R. I. Tobey, J. P. Hill, and X. J. Wang, New J. Phys. 17, 063004 (2015).

${ }^{36}$ F. Fu, S. Liu, P. Zhu, D. Xiang, J. Zhang, and J. Cao, Rev. Sci. Instrum. 85, 083701 (2014).

${ }^{37}$ D. Filippetto, M. M. Munoz, H. Qian, F. Sannibale, R. Wells, W. Wan, and M. Zolotorev, in Proceedings of IPAC14, Dresden, Germany, 2014, http:// www.jacow.org, p. MOPRI053.

${ }^{38}$ See https://www6.slac.stanford.edu/files/Strategic_Plan_2014.pdf for SLAC Strategic Plan, 2014.

${ }^{39}$ R. Akre et al., Phys. Rev. Spec. Top.-Accel. Beams 11, 030703 (2008).

${ }^{40}$ D. H. Dowell, E. Jongewaard, J. Lewandowski, C. Limborg-Deprey, Z. Li, J. Schmerge, A. Vlieks, J. Wang, and L. Xiao, in ICFA Beam Dynamics Newsletter No. 46, edited by M. A. Furman and W. Chou (International Committee for Future Accelerators, 2008), pp. 162-192.

${ }^{41}$ F. J. Decker, A. Krasnykh, B. Morris, and M. Nguyen, in 2012 IEEE International Power Modulator and High Voltage Conference (IPMHVC) (IEEE, San Diego, CA, USA, 2012), pp. 695-699.

${ }^{42}$ R. Akre, D. Dowell, P. Emma, J. Frisch, B. Hong, K. Kotturi, P. Krejcik, J. Wu, and J. Byrd, in Proceedings of PAC07 (IEEE, Albuquerque, NM, USA, 2007), p. WEPMS036.

${ }^{43}$ B. Hong, R. Akre, and V. Pacak, in Proceedings of IPAC'10, Kyoto, Japan, 2010, http://www.jacow.org, p. TUPEA061.

${ }^{44}$ C. Rivetta, R. Akre, P. Cutino, J. Frisch, and K. Kotturi, in Proceedings of PAC07 (IEEE, Albuquerque, NM, USA, 2007), p. MOPAS061.

${ }^{45} \mathrm{~K}$. Gumerlock et al., in Proceedings of FEL2014, Basel, Switzerland, 2014, http://www.jacow.org, p. THP080.

${ }^{46}$ H. Park, Z. Hao, X. Wang, S. Nie, R. Clinite, and J. Cao, Rev. Sci. Instrum. 76, 083905 (2005).

${ }^{47}$ J. R. Dwyer, C. T. Hebeisen, R. Ernstorfer, M. Harb, V. B. Deyirmenjian, R. E. Jordan, and R. J. D. Miller, Philos. Trans. R. Soc., A 364, 741 (2006).

${ }^{48}$ C. M. Scoby, R. K. Li, and P. Musumeci, Ultramicroscopy 127, 14 (2013).

${ }^{49}$ C. M. Scoby, R. K. Li, E. Threlkeld, H. To, and P. Musumeci, Appl. Phys. Lett. 102, 023506 (2013).

${ }^{50}$ M. Gulde, S. Schweda, G. Storeck, M. Maiti, H. K. Yu, A. M. Wodtke, S. Schäfer, and C. Ropers, Science 345, 200 (2014).

${ }^{51}$ See http://www.andor.com/ for information about Andor iXon Ultra 888 EMCCD.

${ }^{52}$ R. K. Li and C. X. Tang, Nucl. Instrum. Methods Phys. Res., Sect. A 605 , 243 (2009).

${ }^{53}$ X. J. Wang, X. Qiu, and I. Ben-Zvi, Phys. Rev. E 54, R3121(R) (1996).

${ }^{54}$ K. Sokolowski-Tinten et al., Nature 422, 287 (2003).

${ }^{55}$ D. M. Fritz, Science 315, 633 (2007).

${ }^{56}$ M. Beye et al., Appl. Phys. Lett. 100, 121108 (2012).

${ }^{57}$ M. Harmand et al., Nat. Photonics 7, 215 (2013).

${ }^{58}$ R. Riedel et al., Nat. Commun. 4, 1731 (2013).

${ }^{59}$ N. Hartman et al., Nat. Photonics 8, 706 (2014).

${ }^{60} \mathrm{D}$. Cesar et al., "Ultrafast gating of a mid-infrared laser pulse by a sub-pC relativistic electron beam" (unpublished).

${ }^{61}$ K. Floettmann, Nucl. Instrum. Methods Phys. Res., Sect. A 740, 34 (2014).

${ }^{62}$ I. Nozawa et al., Phys. Rev. Spec. Top.-Accel. Beams 17, 072803 (2014).

${ }^{63}$ X. Lu, C. Tang, R. Li, H. To, G. Andonian, and P. Musumeci, Phys. Rev. Spec. Top.-Accel. Beams 18, 032802 (2015).

${ }^{64}$ A. H. Reid, "Development of nano-ultrafast electron diffraction at SLAC," SLAC LDRD Proposal, 2014.

${ }^{65}$ N. de Jonge and F. M. Ross, Nat. Nanotechnol. 6, 695 (2011).

${ }^{66} \mathrm{Z}$. Wu, A. S. Fisher, J. Goodfellow, M. Fuchs, D. Daranciang, M. Hogan, H. Loos, and A. Lindenberg, Rev. Sci. Instrum. 84, 022701 (2013). 